\title{
Mössbauer Effect in Frozen Solutions of Nitroprusside
}

Cite as: J. Chem. Phys. 50, 3127 (1969); https://doi.org/10.1063/1.1671525

Submitted: 12 November 1968 . Published Online: 19 December 2003

W. A. Mundt, and T. Sonnino

\section{ARTICLES YOU MAY BE INTERESTED IN}

Mössbauer Hyperfine Interactions in Sodium Nitroprusside Single Crystals

The Journal of Chemical Physics 47, 382 (1967); https://doi.org/10.1063/1.1711904

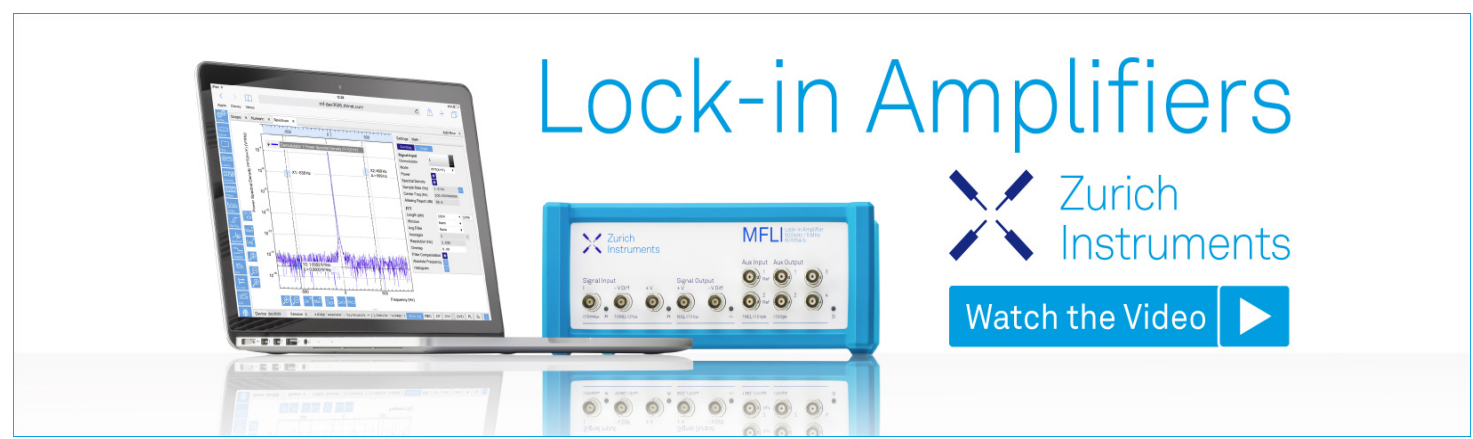


TABLE I. Observed lines of the $c^{3} \amalg-X^{1} \Sigma^{+}$transition.

\begin{tabular}{|c|c|c|c|}
\hline $\boldsymbol{J}$ & $P(J)$ & $Q(J)$ & $R(J)$ \\
\hline 1 & & & 92084.99 \\
\hline 2 & 92068.41 & & 089.15 \\
\hline 3 & 065.81 & & 093.31 \\
\hline 4 & 062.51 & & 097.36 \\
\hline 5 & 058.16 & & 101.73 \\
\hline 6 & 055.00 & & 105.88 \\
\hline 7 & 052.14 & & 110.54 \\
\hline 8 & 047.96 & 92078.97 & 115.07 \\
\hline 9 & 045.09 & 080.21 & 119.89 \\
\hline 10 & 042.95 & 081.02 & 124.59 \\
\hline 11 & 039.73 & 081.86 & 129.45 \\
\hline 12 & 036.36 & 082.79 & \\
\hline 13 & 032.99 & 083.95 & \\
\hline 14 & 030.11 & 084.99 & \\
\hline 15 & & 086.24 & \\
\hline 16 & & 087.63 & \\
\hline 17 & & 089.15 & \\
\hline 18 & & 090.38 & \\
\hline 19 & & 091.91 & \\
\hline
\end{tabular}

of figure). Branches of the new transition are illustrated at the top of the figure.

A condensed $Q$ branch is consistent with either a II-1 $\Sigma^{+}, \Delta^{-1} \Sigma^{+}$, or ${ }^{3} \Sigma^{-1} \boldsymbol{\Sigma}^{+}$transition but not with a ${ }^{3} \Sigma^{+-1} \Sigma^{+}$transition. For a ${ }^{3} \Sigma^{-}$upper state, three $Q$-type branches are expected, but no $P$ or $R$ branches are permitted. Therefore, the upper state of this transition must be $\Pi$ or $\Delta$ in disagreement with the previous assignment ${ }^{2,5}$ (unless two electronic states in the $\mathrm{CO}$ molecule occur within $\sim 0.5 \mathrm{~cm}^{-1}$ of each other which is extremely unlikely).

On the basis of the strength of this transition in absorption, the near-ultraviolet transition in emission, ${ }^{5}$ theoretical calculations ${ }^{10}$ which correlate very well with other low-lying Rydberg states in $\mathrm{CO},{ }^{7,9}$ and recent high-angle electron scattering observations, ${ }^{11}$ it is most probable that the $c$ state is a ${ }^{3} \Pi$ state. As stated earlier, ${ }^{9}$ reanalysis of Gerö's data ${ }^{5}$ does not preclude the assignment of the near-ultraviolet transition to a ${ }^{3} \Pi-{ }^{-3} \Pi$ transition; however, the apparent resolution of the older data is too poor to make a definite decision.

If the upper state is ${ }^{3} \mathrm{II}$ we have observed a transition to only one of the triplet components, unless the ${ }^{3} \Pi$ state is pure Hunds case " $b$ " in which there would be no spin splitting. Because of the uncertainty in the $\Lambda$-type doubling in the upper state, the $Q(J)$ lines may be in error by 2 or more $J$ numbers. The $Q(J)$ lines were assigned to minimize the splitting between the $\Pi^{+}$and $\Pi^{-}$components in the upper state.

Observed band lines are tabulated in Table I. Values of $B^{+}=1.963 \mathrm{~cm}^{-1}$ and $\nu_{0}=92076.9 \mathrm{~cm}^{-1}$ have been derived from a least-squares fit of the data. Within the limits of error for the two sets of data, these quantities are in good agreement with those determined from the near-ultraviolet data of Gerö. ${ }^{5}$

1 P. H. Krupenie, Natl. Std. Ref. Data Ser., Natl. Bur. Std. (U.S.) NSRDS-NBS 5 (1966).

2 R. Schmid and L. Gerö, Nature 139, 928 (1937).

${ }^{8}$ L. Gerö, G. Herzberg, and R. Schmid, Phys. Rev. 52, 467 (1937).

${ }^{4}$ G. H. Dieke and J. W. Mauchly, Phys. Rev. 43, 12 (1933).

${ }^{5}$ L. Gerö, Z. Physik 109, 210 (1938).

- P. G. Wilkinson and E. T. Byram, Appl. Opt. 5, 581 (1965).

${ }^{7}$ S. G. Tilford, J. T. Vanderslice, and P. G. Wilkinson, Can. J. Phys. 43, 450 (1965).

S. G. Tilford and J. T. Vanderslice, J. Mol. Spectry. 26, 419 (1968).

9 J. E. Hesser and K. Dressler, J. Chem. Phys. 45, 3149 (1966). ${ }^{10} \mathrm{H}$. Lefebvre-Brion, C. M. Moser, and R. K. Nesbet, J. Mol. Spectry, 13, 418 (1964).

${ }^{11}$ H. H. Brongersma and L. J. Oosterhoff, Chem. Phys. Letters 1, 169 (1967).

\section{Mössbauer Effect in Frozen Solutions of Nitroprusside*}

\author{
W. A. Mundt and T. Sonnino $\dagger$ \\ Instituto de Fisica, Universidade Federal do Rio Grande do Sul, \\ Pôrto Alegre, Brasil
}

(Received 12 November 1968)

The results of Mössbauer-effect experiments are very often interpreted in terms of molecular electronic structure, but if the Mössbauer nucleus is bound in a covalent charged complex, the effects of the external ions are ignored. The lattice effects on the electronic structure are also ignored in the case of molecular crystals. Frozen solutions can be used to study those effects.

In recent years the Mössbauer effect has been applied to frozen aqueous solutions of some inorganic iron salts, ${ }^{1,2}$ in order to study the crystalline arrangement of water molecules and its effect, at temperatures between about $-190^{\circ}-0^{\circ} \mathrm{C}$. The effects of the lattice have been demonstrated to be quite important in the case of molecular $\mathrm{I}_{2}$ using ${ }^{129} \mathrm{I}$ in the inert organic solvents. ${ }^{3}$ Our purpose was to follow another line, investigating iron complexes, in which the influence of water dipoles in the frozen solution would be presumably negligible and certainly the interaction solute-solvent smaller than the effects that are to be measured. ${ }^{2}$

In this Note we report an experiment carried out to test the effect of ions external to the complex. We have chosen sodium nitroprusside

$$
\mathrm{Na}_{2} \mathrm{Fe}(\mathrm{CN})_{5}(\mathrm{NO}) \cdot 2 \mathrm{H}_{2} \mathrm{O} \text {, }
$$




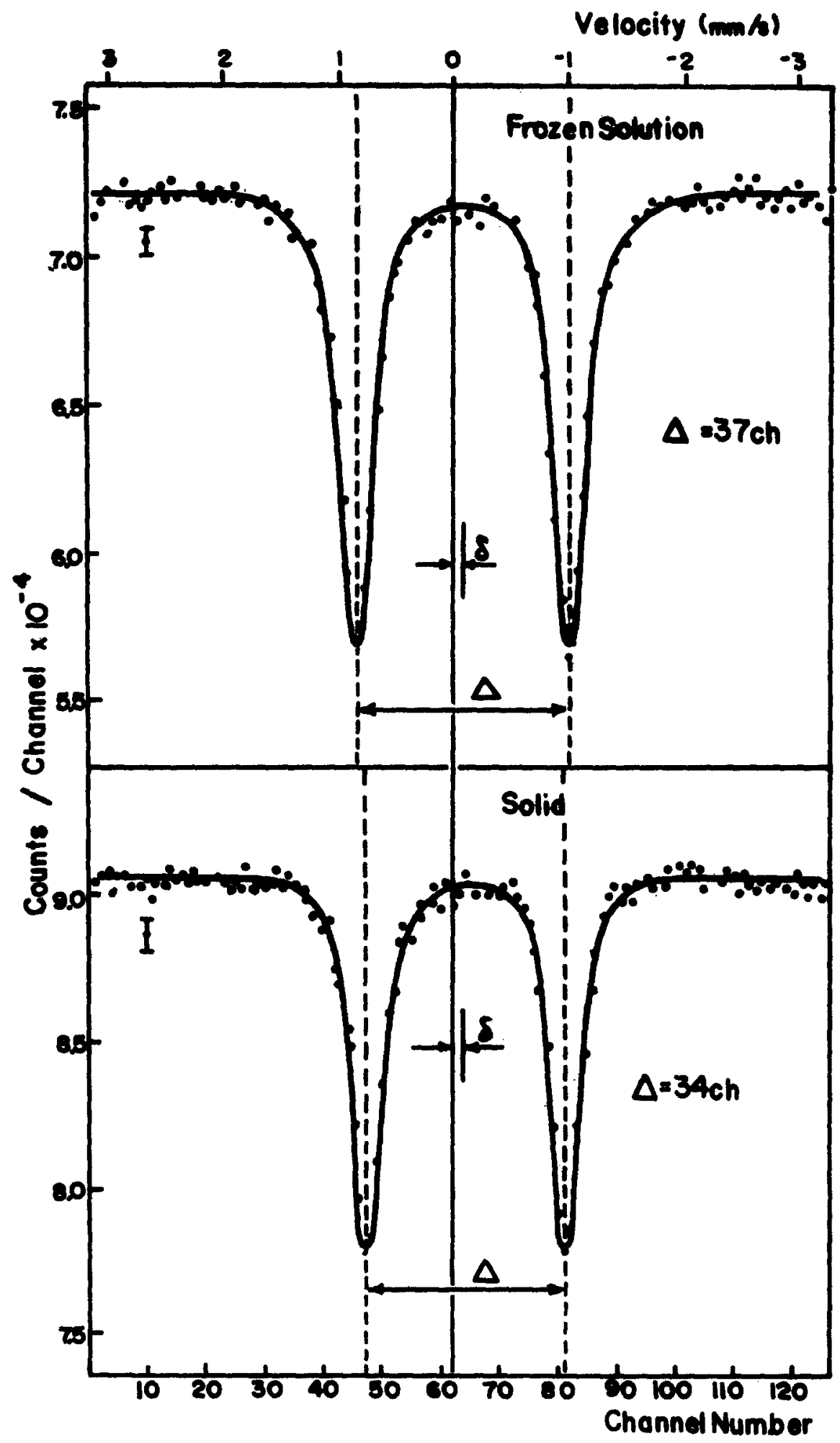

FIG. 1. The Mössbauer effect of aqueous frozen solution (upper) and of solid (lower) nitroprusside, both at liquid-nitrogen temperature.

whose properties have been exhaustively studied ${ }^{4}$ and the results interpreted using electronic structure calculations. ${ }^{5}$ The nitroprusside molecule has the structure of a distorted octahedron, the iron atom occupying nearly the center and four $\mathrm{CN}$ groups being equatorial and the fifth $\mathrm{CN}$ group colinear with the $\mathrm{Fe}$ nucleus and NO group on the axial direction.

Aqueous solutions of sodium nitroprusside were prepared with concentrations between $0.05 M$ and $1.4 M$ (saturated solution). 
Solutions in ethyl alcohol with a concentration smaller than $0.05 M$ were also investigated, to test the possible influence of the solvent. These solutions were introduced in an absorber holder with Mylar windows and rapidly cooled with liquid nitrogen. To test the influence of ionic concentration another series of solutions was prepared with a nitroprusside concentration of $0.1 M$ and an $\mathrm{NaCl}$ concentration up to $2 M$. The Mössbauer effect was measured using a constant acceleration setup and a ${ }^{57} \mathrm{Co}$ source in a $\mathrm{Cr}$ matrix. The results were reproducible and are shown in Fig. 1, compared with solid nitroprusside at liquid-nitrogen temperature.

The quadrupole splitting $\Delta=\frac{1}{2} e^{2} q Q$ increased in the frozen solutions to $1.86 \pm 0.03 \mathrm{~mm} / \mathrm{sec}$ as compared to $1.71 \mathrm{~mm} / \mathrm{sec}$ in the solid, independently of concentration and ionic strength. The isomer shift $\delta$ is equal for solid and frozen solutions, except for the secondorder Doppler shift. ${ }^{6}$

The same increase in quadrupole coupling was observed in the alcoholic solution, thus confirming the negligible effect of water dipoles in the behavior of the iron complex.

The difference in $\frac{1}{2} e^{2} q Q$ in the solid and frozen solutions can be explained as an effect of polarization of the cations on the CN ligand groups. Direct effects can be ruled out, as the two $\mathrm{Na}^{+}$ions in the solid are too far away to contribute directly to the electric field gradient.

According to Danon, 4

$$
q=(4 / 7)\left\langle r^{-3}\right\rangle\left(n_{x y}\right)-(2 / 7)\left\langle r^{-3}\right\rangle\left(n_{x z}+n_{y z}\right),
$$

where $n$ is the electron population in the various iron $d$ orbitals. From Ref. 5 the charge distribution of the complex is $(\mathrm{Fe})^{+0.2166}(\mathrm{CN})_{4}^{-2.200}(\mathrm{CN})^{-0.5800}(\mathrm{NO})^{+0.4643}$, where $(\mathrm{CN})$ are the four equatorial ligands in the $x y$ plane, the other (CN) being axially colinear with the NO group in the $z$ direction.

In the solid the two $\mathrm{Na}^{+}$ions will polarize the negative $\mathrm{CN}$ groups, hence also the $\mathrm{Fe}-\mathrm{CN}$ bonds, and affect the electron populations of the iron orbitals. This effect will result in a different contribution of $q$ for axial and equatorial bonds, as lower population in the equatorial bonds $(x y)$ will decrease $q$, the opposite for the axial.

In the solutions, where the complex is presumably surrounded by solvent molecules, this polarization will be removed, and the charge in the $\mathrm{Fe}-\mathrm{CN}$ bond increased. This effect in the four equatorial bonds will result in higher electron population in the $d_{x y}$ orbitals of iron, $\left(n_{x y}\right)$, and hence increase the quadrupole splitting as has been actually found.

\footnotetext{
* Work supported partially by CNPq (Brasil), CPq (UFGGS), Air Force Office of Scientific Research, and I.A.E.A.

$\dagger$ I.A.E.A. Visiting Professor. Permanent address: Soreq Nuclear Research Center, Yavne, Israel.

${ }^{1}$ I. Dezsi, L. Keszthelyi, B. Molnar, and L. Pocs, Proc. Conf. Hyperfine Structure Nucl. Radiations, Asilomar, Calif., 1967, 566 (1968).
}

2 A. J. Nozik and M. Kaplan, J. Chem. Phys. 47, 2960 (1967). ${ }^{3} \mathrm{~S}$. Buckshpan, C. Goldstein, and T. Sonnino 49, 5477 (1968).

${ }^{4}$ J. Danon and L. Iannarella, J. Chem. Phys. 47, 382 (1967) and references cited therein. It is interesting also to remember that nitroprusside has been proposed as a standard material for the Mössbauer effect in ${ }^{57} \mathrm{Fe}$. Intern. At. Energy Agency, Tech. Rept. Ser. 50, 254 (1966).

${ }^{5}$ P. T. Manoharan and H. B. Gray, J. Am. Chem. Soc. 87, 3340 (1965).

${ }^{6}$ W. Kerler and W. Neuwirth, Z. Physik 167, 176 (1962).

\section{Quenching of $\mathbf{N}_{2}\left(A^{8} \Sigma_{u}+\right)$ in High Vibrational Levels by Nitrogen Atoms}

\author{
Mich ael P. Weinreb and Gene G. Mannell a \\ Electronics Research Center, Cambridge, Massachuselts
}

(Received 18 October 1968)

Several researchers ${ }^{1}$ have suggested that the primary process in removing $\mathrm{N}_{2}\left(A^{3} \Sigma_{u}{ }^{+}\right)$in low $(v=0,1)$ vibrational levels from active nitrogen is

$$
\mathrm{N}_{2}\left(A^{8} \Sigma_{u}{ }^{+}\right)+\mathrm{N}\left({ }^{4} S\right) \rightarrow \mathrm{N}_{2}\left(X^{1} \Sigma_{0}{ }^{+}\right)+\mathrm{N},
$$

with a rate coefficient, $k_{1}$, of $5 \times 10^{-11} \mathrm{~cm}^{8} \mathrm{sec}^{-1}$, corresponding almost to the kinetic cross section; Thrush ${ }^{2}$ estimated $k_{1}$ at $5 \times 10^{-12} \mathrm{~cm}^{3} \mathrm{sec}^{-1}$. Also interesting is the value of $k_{1}$ where Reaction (1) involves $\mathrm{N}_{2}\left(A^{3} \Sigma_{u}{ }^{+}\right)$ in high vibrational levels, since, for example, this reaction might interfere with the mechanism suggested by Campbell and Thrush ${ }^{3}$ for populating the $\mathrm{N}_{2}\left(B \Pi_{0}\right)$ state in active nitrogen.

In studies on surface-catalyzed excitation of nitrogen, ${ }^{4}$ we have deduced an upper limit of $5 \times 10^{-13}$ $\mathrm{cm}^{3} \mathrm{sec}^{-1}$ for $k_{1}$ when Reaction (1) involves $\mathrm{N}_{2}\left(A^{3} \Sigma_{u}{ }^{+}\right)$ in high levels.

Surface-catalyzed excitation of nitrogen 4 occurs when a mixed stream of nitrogen atoms and discharged oxygen passes over a nickel, cobalt, or copper surface. A red glow appears in the gas above the surface, its intensity decreasing with increasing height above the surface until it disappears in the background at a height of several centimeters. This glow contains only first positive bands of $\mathrm{N}_{2}$. The most intense bands originate from $v=6$ of the $B^{3} \Pi_{\sigma}$ state, and bands from levels above the eighth are suppressed. The evidence indicates ${ }^{4}$ that nitrogen atoms recombining at the surface form molecules in high $(v \geq 7)$ levels of the $A$ state. These molecules diffuse into the gas and cross into the $B$ state through collisions, i.e., $\mathrm{N}_{2}\left(A^{3} \Sigma_{u}{ }^{+}\right)+\mathrm{M} \rightarrow \mathrm{N}_{2}\left(B^{3} \Pi_{\sigma}\right)+\mathrm{M}$. These $B$-state molecules radiate immediately in transitions to low levels of the $A$ state.

In this paper we use measurements of the intensity of this glow to determine $k_{1}$. Above the surface, the steady-state concentration of $A$-state molecules in high levels is mainly governed by three processes: the diffusion of these $A$-state molecules from the surface where they were formed; the $A$ - to $B$-state crossing; and Reaction (1). Solution of the rate equation yields 